\title{
1 The microtubule plus-end tracking protein Bik1 is required for
}

\section{2 chromosome congression}

4 Alexander Julner ${ }^{1}$, Marjan Abbasi ${ }^{1}$, and Victoria Menéndez-Benito ${ }^{1,2}$

1 Department of Biosciences and Nutrition, Karolinska Institutet, SE-141 83, Huddinge, Sweden.

2 Correspondence: victoria.menendez.benito@ki.se

7

8

RUNNING HEAD: Bik1 controls chromosome congression

10

ABBREVIATIONS: Adenosine triphosphate (ATP); nuclear export signal (NES); spindle pole body (SPB);

12 microtubule plus-end tracking proteins (+TIPs); superfolder GFP (sfGFP); bimolecular fluorescence

13 complementation (BiFC).

14

15 Number of characters: 16815 


\section{Abstract}

17 During mitosis, sister chromatids congress on both sides of the spindle equator to facilitate the

18 correct partitioning of the genomic material. Chromosome congression requires a finely tuned

19 control of microtubule dynamics by the kinesin motor proteins. In Saccharomyces cerevisiae, the

20 kinesin proteins Cin8, Kip1, and Kip3 have a pivotal role in chromosome congression. It has been

21 hypothesized that additional proteins that modulate microtubule dynamics are also involved. Here,

22 we show that the microtubule plus-end tracking protein Bik1 - the budding yeast ortholog of CLIP-

$23170-$ is essential for chromosome congression. We find that nuclear Bik1 localizes to the

24 kinetochores in a cell-cycle-dependent manner. Disrupting the nuclear pool of Bik1 with a nuclear

25 export signal (Bik1-NES) leads to a slower cell cycle progression characterized by a delayed

26 metaphase-anaphase transition. Bik1-NES cells have mispositioned kinetochores along the spindle in

27 metaphase. Furthermore, using proximity-dependent methods, we identify Cin8 as an interaction

28 partner of Bik1. Deleting CIN8 reduces the amount of Bik1 at the spindle. In contrast, Cin8 retains its

29 typical bilobed distribution in the Bik1-NES mutant and does not localize to the unclustered

30 kinetochores. Thus, we propose that Bik1 functions with Cin8 to regulate kinetochore-microtubule

31 dynamics for correct kinetochore positioning and chromosome congression.

32

33 Keywords: mitosis, kinetochore, chromosome, kinesins, genetic stability. 
Cell division relies on the accurate segregation of sister chromatids to generate functional daughter cells. Before segregation, sister chromatids are positioned at the spindle equator through a process known as chromosome congression that culminates with the metaphase plate formation in metazoans (reviewed in (Maiato et al., 2017)). The establishment of a metaphase plate forces all the chromosomes to segregate from the same starting position, thereby contributing to faithful segregation. Despite the relevance of chromosome congression, its molecular mechanisms remain unclear.

The budding yeast Saccharomyces cerevisiae is an appealing model organism to study spindle dynamics because it is amenable to genetic engineering and has a relatively simple spindle. Budding yeast has 16 chromosomes. After duplication, each sister chromatid is linked by a kinetochore to a single kinetochore microtubule emanating from the spindle pole body (SPB), the yeast centrosome (Winey \& O'Toole, 2001). Each SPB also gives rise to a few interpolar microtubules (starting with around six and ending with two interpolar microtubules from each SPB), with plus-ends that overlap at the middle of the spindle (O'Toole et al., 1999; Peterson \& Ris, 1976; Winey et al., 1995). In prometaphase, kinetochores congress in two opposite clusters near the spindle equator, equidistant from the SPBs. As in metazoan cells, this appears to occur prior to biorientation of sister chromatids (Marco et al., 2013).

Several studies have shown that kinetochore microtubules are regulated in a length-dependent manner (Gardner et al., 2005; Pearson et al., 2006; Sprague et al., 2003). Short microtubules tend to grow, whereas long microtubules that extend towards the spindle equator are destabilized and depolymerize. As sister chromatids are held together by cohesin, depolymerization of kinetochore microtubules generates tension, mediated by the Ndc80 and Dam1 complexes' ability to track the dynamic tips of the kinetochore microtubules (Powers et al., 2009; Suzuki et al., 2016). 
61 Kinesin motors - proteins that move along microtubules powered by the hydrolysis of adenosine

62 triphosphate (ATP) - are involved in chromosome congression in several organisms (see Maiato et

63 al., 2017, Table 1). In budding yeast, the deletion of the kinesins Cin8, Kip3, and, to a lesser extent,

64 Kip1, results in declustering of kinetochores (Gardner et al., 2008; Marco et al., 2013; Tytell \& Sorger,

2006; Wargacki et al., 2010). Cin8 and Kip3 have been shown to act as length-dependent microtubule depolymerases, promoting catastrophe of long microtubules (Gardner et al., 2008; Su et al., 2011; Varga et al., 2006).

It has long been speculated that other microtubule-associated proteins could play a role in (+TIPs) are interesting candidates, because they accumulate at kinetochore microtubule plus-ends and regulate their dynamics (reviewed in Akhmanova \& Steinmetz, 2010). Bik1, the yeast ortholog of CLIP-170, is a +TIP protein that exists in cytoplasmic and nuclear pools within the cell (Carvalho et al., 2004). The cytoplasmic pool is important for mitotic spindle positioning through both the Kar9 and the dynein pathways (reviewed in Miller et al., 2006). First, Bik1 promotes phosphorylation of Kar9 for asymmetric localization at the old SPB (Moore et al., 2006). Kar9, together with the +TIP protein Bim1 and the actin-guided myosin motor protein Myo2, mediates the capture of astral microtubules at the bud cortex, contributing to spindle alignment and nuclear migration (Xiang, 2018). During anaphase, Bik1 is transported by the kinesin Kip2 to the plus-ends of astral microtubules, where it facilitates the recruitment of dynein (Carvalho et al., 2004; Roberts et al., 2014). Dynein is a minus end-directed motor and will pull the spindle resulting in nuclear migration to the bud.

The nuclear pool of Bik1 is associated with the spindle at SPBs and kinetochores (Carvalho et al., 84 2004; Lin et al., 2001), but its function is not well understood. Early studies found that deleting BIK1 results in cells with short or non-detectable astral microtubules (Berlin et al., 1990). In contrast, cells overexpressing Bik1 display short spindle microtubules and long astral microtubules. In this study, 

after which it instead is localized to interpolar microtubules. progression.

\section{Results and discussion}

\section{Bik1 localizes to kinetochores in a cell cycle-dependent manner}

we investigate the nuclear function of Bik1 and its role in chromosome congression and cell cycle

To better understand the nuclear pool of Bik1, we first explored the localization of the protein at different stages of the cell cycle. We used cells expressing Bik1-superfolder GFP (sfGFP), mTurquoiseTub1 (alpha tubulin, microtubule marker), and either Ndc80-tagRFPT (kinetochore marker) or Spc42tagRFPT (SPB marker) (Figure 1A). In metaphase cells, nuclear Bik1 was primarily found between SPBs and overlapping with kinetochores. This bilobed kinetochore-associated distribution has also been reported for the kinesins Cin8, Kip1, and Kip3 (Gardner et al., 2008; Tytell \& Sorger, 2006; Wargacki et al., 2010). Anaphase cells, on the other hand, displayed nuclear Bik1 concentrated in patches along interpolar microtubules, again similar to what is reported for kinesins Cin8, Kip1, and Kip3. Cytoplasmic Bik1, meanwhile, was localized to astral microtubule tips at the cell cortex.

\section{To confirm the metaphase association with kinetochores, we used cells expressing the APC/C co-} activator Cdc20 under the control of the galactose inducible GAL1 promoter. Shifting the carbon source from galactose to glucose represses the expression of $\mathrm{CDC} 20$ and thereby arrests the cells at the metaphase-to-anaphase transition. Bik1-sfGFP was localized at kinetochore clusters in metaphase arrested cells (Figure 1B), confirming what we observed in cycling cells with short spindles. In summary, we conclude that Bik1 associates with kinetochores from G1 until metaphase, 


\section{Nuclear Bik1 is needed for timely cell cycle progression}

111 Bik1 in the cytoplasm localizes to astral microtubules and plays an important role in mitotic spindle

112 positioning (Carvalho et al., 2004; Moore et al., 2006). To disrupt the kinetochore function of Bik1

113 while maintaining its cytoplasmic function, we fused a strong nuclear export consensus sequence

114 (NES) (Kosugi et al., 2008) to the C-terminal end of Bik1-sfGFP or Bik1-5xFlag (collectively referred to as Bik1-NES, Figure 1C). We imaged Bik1-NES cells expressing mTurquoise-Tub1, and Nc80-tagRFPT and confirmed that the mutant indeed excludes Bik1 from the nucleus while maintaining its localization at astral microtubules (Figure 1D). To quantify the effectiveness of the NES, we followed the localization of Bik1 in Bik1-sfGFP and Bik1-sfGFP-NES cells expressing mTurquoise-Tub1. We

119 synchronized the cells in G1 with $\alpha$-factor, released them in fresh media, and fixed cells at 10-minute

120 intervals from release. We then measured Bik1 intensity along the spindle in metaphase cells and

121 found that, as expected, Bik1-NES cells have significantly less sfGFP signal at the spindle microtubules 122 than Bik1 cells (Figure 1E).

124 We next assessed cell cycle progression in the absence of nuclear Bik1. We synchronized Bik1-sfGFP 125 and Bik1-sfGFP-NES cells in G1 with $\alpha$-factor and scored the number of cells in G1/S, metaphase, or 126 anaphase according to the spindle length (mRuby2-Tub1) (Figure 2A). The strain expressing Bik1sfGFP had a sharp peak of metaphase cells at $40 \mathrm{~min}$ and a high number of cells with long spindles

128 50-60 min after the release. Bik1-sfGFP-NES cells, by contrast, displayed a broader peak of metaphase cells that spans between 40-60 min post-release and a 10 min delay in the anaphase

130 peak.

132 We then examined Bik1-sfGFP and Bik1-sfGFP-NES cells after G1 synchronization with $\alpha$-factor by

133 time-lapse imaging (Figure 2B-E). In the Bik1-sfGFP strain, Bik1 was localized to the kinetochores

134 from G1 through metaphase. When the spindle elongates in anaphase, Bik1 was no longer associated 135 with the kinetochores and was found in patches along the interpolar microtubules instead (Figure 
136 2B-C), as observed in fixed cells (Figure $1 \mathrm{~A}$ ). The fluorescence intensity of Bik1-sfGFP at kinetochores

137 was highest at metaphase when kinetochores cluster and reduced to near background levels in

138 anaphase (Figure 2D). In agreement with the cell-cycle analysis from fixed cells, we observed a slight

139 delay in cell cycle progression in Bik1-NES cells (Figure 2B, arrows). Our quantifications showed that

140 the time from the appearance of two kinetochore clusters to their segregation into mother and

141 daughter cells is approximately 10 min longer in Bik1-NES cells (Figure 2E). Interestingly, we

142 occasionally observed all kinetochores of short spindles transferred to the daughter cell in the NES

143 mutant strain before elongation occurred (Figure 2 B, $15 \mathrm{~min}$ and 25-30 $\mathrm{min}$ ). We interpret this as a

144 secondary effect of the prolonged metaphase of the mutant.

Cells lacking nuclear Bik1 display aberrant kinetochore positioning

147 Because Bik1 localizes near the kinetochores in metaphase, we hypothesized that the prolonged

148 metaphase of Bik1-NES cells might be due to a dysregulation in kinetochore clustering. To test this

149 hypothesis, we analyzed the distribution of the kinetochore component Ndc80-sfGFP relative to the

150 SPBs (Spc42-tagRFPT) in cells expressing either Bik1-5xFlag or Bik1-5xFlag-NES arrested in metaphase

151 (PGal1-Cdc20) (Figure 3). While Bik1-5xFlag cells showed typical Ndc80 clusters, we observed cells

152 with long stretches of Ndc80 in the Bik1-5xFlag-NES mutant (Figures 3A-B). The unclustered

153 kinetochore phenotype was observed in $60 \%$ of the Bik1-NES metaphase cells (Figure 3C). Line scan

154 analysis of the Ndc80-sfGFP fluorescence relative to the SPBs showed that Bik1-NES cells have a

155 flattened distribution of $\mathrm{Ndc} 80$ with kinetochores mispositioned towards the spindle equator (Figure $1563 \mathrm{D})$.

158 Bik1 has previously been suggested to contribute to the kinetochore-microtubule attachment. Bik1 is

159 needed to nucleate microtubules that capture kinetochores after centromere re-activation (Tanaka

160 et al., 2005). Furthermore, a binding partner of Bik1, the +TIP Bim1, was recently shown to promote

161 in vitro oligomerization of Dam1 complexes- a major component of the yeast outer kinetochore 
162 (Dudziak et al., 2021). Therefore, Bik1's function in kinetochore end-on attachment could contribute

163 to the cell cycle delay we observe. Yet, we did not detect unattached kinetochores in the Bik1-NES

164 mutant. Instead, the kinetochores are aligned in the spindle but appear unclustered during

165 metaphase.

166

Previous studies have shown that the kinesins Cin8 and to a lesser extent Kip1 and Kip3 are required for yeast chromosome congression (Gardner et al., 2008; Tytell \& Sorger, 2006; Wargacki et al., 2010). However, the deletion of each kinesin results in slightly different types of clustering defects. In the cin8 $\Delta$ mutant, the kinetochores are positioned closer to the spindle equator (Gardner et al.,

171 2008; Tytell \& Sorger, 2006; Wargacki et al., 2010). The kip1 $\Delta$ mutant has a similar, but weaker, 172 phenotype (Gardner et al., 2008; Tytell \& Sorger, 2006). Conversely, Ndc80 clusters in kip3 $\Delta$ cells 173 appear closer towards the spindle poles (Wargacki et al., 2010). We found that the Ndc80 foci in the 174 Bik1-NES mutant appear shifted towards the spindle equator (Figure 3D), as has been reported for 175 cin8 $\Delta$ cells.

\section{Bik1 interacts with Cin8 at the spindle}

178 To investigate Bik1's association with kinesins, we performed single-step affinity purification of Bik1Flag in metaphase arrested cells, followed by mass spectrometry analysis. Bik1-Flag co-purified with the yeast kinesins Kip2, Cin8, Kip1, Kip3 and Kar3 (Table 1). Notably, the kinesin Cin8 appeared with a similar coverage as the known interactor Kip2 (Supplementary data S1). The defect of Bik1-NES cells in kinetochore clustering shown in Figure 3A phenocopies CIN8 deletion

184 (Gardner et al., 2008; Tytell \& Sorger, 2006; Wargacki et al., 2010). Therefore, we decided to study

185 the Bik1-Cin8 interaction in more detail. We investigated whether Cin8 and Bik1 interact in vivo 186 using proximity-dependent biotin identification (TurbolD), a proximity labelling assay that can detect 187 transient or weak protein-protein interactions (Roux et al., 2012). We fused TurbolD, a mutated 
version of a bacterial biotin ligase BirA (Branon et al., 2018; Larochelle et al., 2019), to the C-terminus of Bik1 (Figure 4A). We then Flag-tagged Cin8 or Kip2, a kinesin that interacts with Bik1 at astral microtubules (Carvalho et al., 2004). Both Cin8 and Kip2 were recovered after streptavidin pulldown when expressed in Bik1-TurbolD cells, indicating that Bik1 and Cin8 are in proximity in vivo (Figure 4B).

We next looked at the interaction between Bik1 and Cin8 in individual cells by bimolecular fluorescence complementation (BiFC) (Sung \& Huh, 2007). Briefly, an N-terminal fragment of the yellow fluorescent protein Venus was fused to the C-terminus of Bik1, and a complimentary Cterminal Venus fragment was fused to the C-terminus of Cin8. We detected BiFC signal at the spindle, predominantly in metaphase and anaphase cells (Figure 4 C-D). Interestingly, the BiFC signal in metaphase cells appeared as 1-2 dots at the spindle, while the signal in anaphase cells appeared as a single dot around the spindle midzone (Figure $4 \mathrm{C}$ ).

We then investigated the dependence of Cin8 and Bik1 to localize to the spindle. In the Bik1-NES mutant, Cin8 still forms two foci between SPBs and kinetochores (Figure 5A and B), even in cells with unclustered kinetochores. This result was surprising as Cin8 has been shown to bind kinetochores directly (Suzuki et al., 2018). Linescan analysis of Cin8-sfGFP localization in relation to SPBs showed that Cin8 localization is unaltered in the absence of nuclear Bik1 (Figure 5B). Deletion of CIN8, on the other hand, resulted in a significantly reduced Bik1 intensity at the spindle (Figure 5C-D). Overall, 208 these data suggest that a portion of nuclear Bik1 associates with and is dependent on Cin8 to bind 209 the spindle.

211 The reduced Bik1 recruitment to the spindle and kinetochores explains why Bik1 cannot compensate 212 for the lack of Cin8, which is known to cause severe defects in assembling the mitotic spindle (Hoyt et 
214 congression by depolymerizing kinetochore microtubules in a length-dependent manner (Gardner et

215 al., 2008). Gardner and coworkers proposed that Cin8 binds randomly to kinetochore microtubules

216 and walks towards the plus-end to promote microtubule plus-end disassembly directly. However, in

217 Bik1-NES cells, Cin8 is insufficient to regulate chromosome congression. Moreover, Cin8 does not

218 localize to the unclustered kinetochores in the Bik1-NES mutant. Taken together, these observations

219 suggest that Bik1 is a binding partner of Cin8 that contributes to the disassembly of kinetochore

220 microtubules to achieve chromosome congression at metaphase. There are at least two other

221 examples where plus-end directed kinesin motors interact with Bik1. Kip2 transports Bik1 to astral

222 microtubule plus ends to ensure proper spindle alignment (Carvalho et al., 2004; Caudron et al.,

223 2008). Furthermore, it has been suggested that Bik1 and Kar3 form a complex at the junction of

224 oppositely oriented MTs during mating to induce microtubule depolymerization for nuclear

225 congression to occur (Molk et al., 2006).

227 We propose that Bik1 increases the processivity of Cin8. Bik1 has been suggested to be a processivity

228 factor for the kinesin Kip2 at astral microtubules (Hibbel et al., 2015). Likewise, Bik1 might be needed

229 for Cin8 to reach the plus-ends of long kinetochore microtubules that extend beyond bundled

230 kinetochore microtubules. Cin8 is a bidirectional kinesin and in vitro studies have shown it

231 preferentially moves in a minus-end directed manner as single motors (Gerson-Gurwitz et al., 2011;

232 Pandey et al., 2021; Roostalu et al., 2011). Directional switching occurs when Cin8 motors are

233 clustered (Pandey et al., 2021; Roostalu et al., 2011), and other kinesin-5 motors switch directionality

234 in response to steric crowding on MTs (Britto et al., 2016). Loss of nuclear Bik1 could therefore lead

235 to ineffective plus-end directed movement of Cin8 on long kinetochore microtubules and failure to

236 cluster kinetochores at metaphase. This is supported by the observation that Cin8 is not localized

237 along rod-like distribution of Ndc80 in the Bik1-NES mutant. 


\section{Materials and Methods}

\section{Yeast strains and plasmids}

All S. cerevisiae strains used in this study are derivatives of BY4741 and are listed in Table S1. The epitope tagged alleles (sfGFP and tagRFPT), TurbolD-3xmyc and BiFC were constructed at the endogenous loci by standard PCR-based integration as described in (Longtine et al., 1998) and confirmed by PCR, sequencing and microscopy. The plasmids used to generate the strains are listed in Table S2. Strains with fluorescently tagged Tub1 were constructed as described in (Markus et al., 2015). Briefly, the listed plasmids were linearized using BsaBI (New England Biolabs, R0537S) and integrated at the endogenous TUB1 locus by homologous recombination.

\section{Growth conditions}

Cells were grown at $30^{\circ} \mathrm{C}$ in YEP ( $1 \%$ yeast extract, $2 \%$ bacto-peptone) medium supplemented with $2 \%$ glucose (YEPD) unless otherwise stated. For G1 arrest, overnight cultures were diluted to $\mathrm{OD}_{600}=$ 0.1 in fresh media, grown for 2.5 hours and arrested by adding $3 \mu \mathrm{g} / \mathrm{ml} \mathrm{a-factor}$ (Sigma, custom peptide WHWLQLKPGQPMY) every $60 \mathrm{~min}$ for $120 \mathrm{~min}$. Cells were released by washing away a-factor with $1 x$ culture volume of YEPD and resuspending in $1 x$ culture volume of YEPD, and further grown at $30^{\circ} \mathrm{C}$. For metaphase arrest, PGal1-Cdc20 cells were grown in YEP supplemented with $2 \%$ raffinose and $2 \%$ galactose, and switched to YEPD for $120 \mathrm{~min}$.

\section{Fluorescence imaging}

Cells were fixed in $4 \%$ formaldehyde on ice for 60 minutes, washed with PBS and stored in PBS at $4^{\circ} \mathrm{C}$ until imaging. Cells were imaged in concanavalin A-coated 96 well glass bottom plates. Images were acquired using a Nikon Ti microscope, equipped with a Zyla 4.2+ sCMOS camera and a 60x water immersion objective (NA 1.2). Z-stack images were captured for fixed cells, as described in the figure 
262 legends. Images for BiFC and immunofluorescence were acquired using a Nikon Ti2 microscope,

263 equipped with a Prime BSI sCMOS camera and a 60x water immersion objective (NA 1.2).

266 Cells were arrested in G1 with as a-factor and subsequently released in low-fluorescence media

267 (LFM) containing $1.7 \mathrm{~g} / \mathrm{l}$ yeast nitrogen base without ammonium sulfate, amino acids, folic acid and 268 riboflavin (MP-Biomedicals, 4030-512), and $1 \mathrm{~g} / \mathrm{l}$ monosodium glutamate, supplemented with $2 \%$ 269 glucose and amino acids. Cells were left to settle on the bottom of concanavalin A-coated 96 well 270 glass bottom plates for 20-30 minutes inside the microscope incubator at $30^{\circ} \mathrm{C}$ before being imaged. 271 Images were captured every 5 min for 100-120 min.

274 Fluorescence intensity measurements were made from maximum intensity projections of image z-

275 stacks using Fidi (Schindelin et al., 2012). Integrated intensities were measured in a region of interest

276 drawn around spindles/kinetochores. Background correction was done by multiplying the mean

277 fluorescence of regions outside of the nucleus with the measured region's area and subtracting from 278 the measured integrated intensity. Linescan analysis was performed by drawing a line between the 279 centers of opposing SPB foci and measuring the fluorescence intensities along the line. Background 280 correction was done by subtracting the mean fluorescence measured at the cytoplasm. Binned 281 means were calculated for each strain. Cell cycle analyses were performed by classifying the cells 282 according to the spindle length, based on the Tub1-tagRFPT marker. (G1/S) spindles $<1 \mu \mathrm{m}$; 283 metaphase $(1-2 \mu \mathrm{m})$, and anaphase $(>2 \mu \mathrm{m})$.

286 PGal1-Cdc20 cells were arrested in metaphase and fixed by adding formaldehyde to $3.7 \%$ (vol/vol) 287 and incubating while shaking at $30^{\circ} \mathrm{C}$ for $30 \mathrm{~min}$. The fixed cells were washed once with PBS and then 
twice with SP buffer (0.1 M KPO4, 1.2 M sorbitol, pH 7.5). The cells were resuspended in $500 \mu \mathrm{LP}$ buffer with $0.1 \% \beta$-mercaptoethanol and $10 \mu \mathrm{l}$ of $20 \mathrm{mg} / \mathrm{ml}$ Zymoylase-20T (Seikagaku). The cells were incubated with soft rotation for $20 \mathrm{~min}$ at room temperature (RT). The resulting spheroplasts were then washed twice in SP buffer and permeabilized in blocking solution (3\% BSA 0.1\% Triton X100 in PBS) for 30 min at RT. The spheroplasts were immunostained in suspension. First, cells were incubated with 1:1000 mouse monoclonal anti-FLAG M2 antibody (Sigma, F1804) in blocking buffer for 1 hour at RT. Cells were washed three times in PBS and then incubated with 1:100 donkey antimouse Alexa Fluor 647 antibody (ThermoFisher, A-31571) for 45 min at RT. Cells were washed three times in PBS, seed in a glass-bottom imaging plate coated with Concanavalin A, and imaged.

Bik1-5xFlag PGal1-Cdc20 cells were arrested in metaphase. Briefly, cells were grown overnight in 50 $\mathrm{ml} \mathrm{YEP}$ with $2 \%$ galactose and $2 \%$ raffinose. Cells were diluted in $2 \mathrm{~L} \mathrm{YEP}$ with $2 \%$ and $2 \%$ raffinose

301 and grown at $30^{\circ} \mathrm{C}$ to $\mathrm{OD}_{600}=1$. Then, cells were spun down and resuspended in $2 \mathrm{LYEPD}$ with $2 \%$

302 glucose and arrested at $30^{\circ} \mathrm{C}$ for $2 \mathrm{~h}$. Cells were spun down at 6000 rom at $4^{\circ} \mathrm{C}$, washed with cold $\mathrm{dH}_{2} \mathrm{O}$, and spun down at $6000 \mathrm{rpm}$ at $4^{\circ} \mathrm{C}$. The cell pellet was diluted in a few drops of lysis buffer (50 $\mathrm{mM}$ Tris, $\mathrm{pH}$ 8.0, $150 \mathrm{mM} \mathrm{NaCl}, 5 \%$ glycerol, $0.1 \%$ Triton X-100). The cell suspension was snap frozen by pipetting the drops directly into liquid nitrogen. The frozen pellets were then lysed using a Freezer/Mill (SPEX ${ }^{\mathrm{TM}}$ sample prep freezer/mill), using 10 rounds consisting of 2 min grinding at 14

$307 \mathrm{rpm}, 2 \mathrm{~min}$ cooling). The yeast pellets were diluted in buffer EB1 (40 mM sodium hepes, $300 \mathrm{mM}$ $308 \mathrm{NaCl}, 0.5 \%$ Triton X-100, $2 \mathrm{mM} \mathrm{MgCl} 2,5 \%$ glycerol and protease and phosphatase inhibitors (1mM 309 PMSF, 1mM leupeptin, 1mM peptasin A, 10mM NaF, 1mM sodium pyrophosphatase, 1mm sodium 310 orthovanadate), and clarified by centrifugation at $10000 \mathrm{~g}$ for $10 \mathrm{~min}$ at $4{ }^{\circ} \mathrm{C}$. The cleared lysate was 311 incubated for 30 min with magnetic Dynabeads (Thermo Fisher Scientific) coupled with anti-FLAG M2 312 antibody (Sigma-Aldrich). The Dynabeads were washed three times with buffer A (25 mM sodium 313 Hepes, 2 mM MgCl $2,0.1 \%$ EDTA, 0.1\% Triton X-100, 5\% glycerol, $150 \mathrm{mM} \mathrm{NaCl}$, protease and 
314 phosphatase inhibitors (1 mM PMSF, $1 \mathrm{mM}$ leupeptin, $1 \mathrm{mM}$ peptasin A, 10mM NaF, $1 \mathrm{mM}$ sodium

315 pyrophosphatase, $1 \mathrm{~mm}$ sodium orthovanadate) and four times with buffer B (25 mM Hepes, pH 8.0,

316 and $150 \mathrm{mM} \mathrm{KCl})$.

317

319 Proteins bound to the Dynabeads were resuspended and washed with $500 \mu \mathrm{L}$ of $50 \mathrm{mM}$ ammonium

320 bicarbonate (AmBic), $\mathrm{pH} 8.0$, twice. After incubation at room temperature for $15 \mathrm{~min}, 50 \mu \mathrm{L}$ of 50

$321 \mathrm{mM}$ AmBic was added. Protein disulfide bonds were reduced with $5 \mu \mathrm{L}$ of $200 \mathrm{mM}$ dithiothreitol at

$32237^{\circ} \mathrm{C}$ for $45 \mathrm{~min}$ and alkylated with $5 \mu \mathrm{L}$ of $600 \mathrm{mM}$ iodoacetamide at room temperature in dark for

$32330 \mathrm{~min}$. Tryptic digestion was started by adding $1 \mu \mathrm{g}$ of trypsin and incubation at $37^{\circ} \mathrm{C}$ overnight,

324 followed by adding another $500 \mathrm{ng}$ of trypsin and incubation at $30^{\circ} \mathrm{C}$ for $3 \mathrm{~h}$. The digestion was

325 stopped with $5 \mu \mathrm{L}$ concentrated formic acid. The samples were then cleaned on a C18 StageTip

326 (Thermo Scientific) and dried using a speedvac (MiVac, Thermo Scientific).

\section{PRLC-MS/MS analysis}

329 Chromatographic separations of peptides were performed on a $50 \mathrm{~cm}$ long C18 EASY-spray column connected to an Easy nanoLC-1000 UPLC system (Thermo Fisher Scientific). The gradient was 4-25\% of solvent B ( $98 \%$ acetonitrile, $0.1 \%$ formic acid) in $50 \mathrm{~min}$ and $25-40 \% \mathrm{~B}$ in $10 \mathrm{~min}$ at a flow rate of

$332300 \mathrm{nl} / \mathrm{min}$. An Orbitrap Fusion mass spectrometer (Thermo Scientific) analyzed the eluted peptides

333 that were ionized in a nano-Easy electrospray source. The survey mass spectrum was acquired at the 334 resolution of 120,000 in the range of $m / z 350-1800 . M S / M S$ data of precursors were obtained with $33530 \%$ normalized collision energy by higher-energy collisional dissociation (HCD) for ions with charge $336 z=2-7$ at a resolution of 30,000 in 3 s cycle time. 
Mascot v 2.5.1 search engine (MatrixScience Ltd., UK) was used for protein identification with the

340 following search parameters: up to two missed cleavage sites for trypsin, peptide mass tolerance 10

$341 \mathrm{ppm}, 0.05 \mathrm{Da}$ for the HCD fragment ions. Carbamidomethylation of cysteine was specified as a fixed

342 modification, whereas oxidation of methionine, deamidation of asparagine and glutamine were

343 defined as variable modifications.

Turbo-ID assay

346 Overnight cultures were diluted to $\mathrm{OD}_{600}=0.2$ in YEPD medium supplemented with $50 \mu \mathrm{M}$ biotin and

347 grown at $30^{\circ} \mathrm{C}$ until they reached $\mathrm{OD}_{600}=0.6(4-5 \mathrm{~h})$. Cells were pelleted and resuspended in RIPA

348 buffer (50 mM Tris-HCl, 150 mM NaCl, 1 mM EDTA, 0.1\% SDS, 1\% Triton X-100, 1.5 mM MgCl2, 0.4\%

349 sodium deoxycholate, $1 \mathrm{mM}$ DTT, $1 \mathrm{mM}$ PMSF, $1 \mathrm{mM}$ leupeptin and1 mM pepstatin A). The cells

350 were lysed using acid-washed glass beads and a bead-beater at $4^{\circ}$. To shear the DNA and RNA, the

351 cell lysate was sonicated, and then clarified by centrifugation. Clarified lysates were incubated with

$352100 \mu$ l of Streptavidin-Sepharose beads (GE Healthcare; $17-5113-01$ ) for $3 \mathrm{~h}$ at $40^{\circ} \mathrm{C}$. The beads were

353 then washed once with wash buffer ( $50 \mathrm{mM}$ Tris-HCl, 2\% SDS) and three times with RIPA buffer. The

354 purified proteins were eluted with $4 \times$ Laemmli buffer containing $25 \mu \mathrm{M}$ biotin and $10 \% \beta$ -

355 mercaptoethanol, for $15 \mathrm{~min}$ at $95^{\circ} \mathrm{C}$. The whole cell extract or pull-down samples were separated by

356 SDS-PAGE, and the proteins were transferred to a PVDF membrane (BioRad) followed by and

357 standard immunoblotting. Primary (monoclonal anti-FLAG M2 antibody (Sigma-Aldrich, F3165,

358 diluted 1:5000); c-Myc rabbit polyclonal antibody (Santa Cruz Biotechnology; sc-789, diluted 1:5000);

359 anti-Pgk1 mouse monoclonal antibody (Invitrogen, 45925, diluted 1:5000), and secondary anti-

360 mouse IgG peroxidase antibody, (Sigma, A9044, diluted 1:50000) were used. HRP conjugated

361 secondary antibodies were detected with Femto ECL (ThermoFisher Scientific). 


\section{Acknowledgements}

364 We thank C. Björkegren (Karolinska Institutet, Sweden) for reagents, and C. Boone and Z. Li for help

365 with construction of deletion mutants and critical reading of this manuscript. Microscopy was

366 performed at the Live Cell Imaging Core facility/Nikon Center of Excellence, at the Karolinska

367 Institutet, supported by grants from the Swedish Research Council, KI infrastructure, and Centre for

368 Innovative Medicine. The Biolmage Informatics Facility at SciLifeLab, National Microscopy

369 Infrastructure (VR-RFI 2019-00217), and recipient of the Chan-Zuckerberg Initiative, assisted in the

370 image analysis. Protein identification and quantification were carried out by the Proteomics

371 Biomedicum core facility, Karolinska Institutet. This work was supported by grants from the Swedish

372 Council (VR-NT 2017-04536), and STINT (Mobility Grant for Internationalization, MG2019-8484) to

373 V.M.B. The authors declare no competing financial interests.

\section{References}

Akhmanova, A., \& Steinmetz, M. O. (2010). Microtubule +TIPs at a glance. In Journal of Cell Science (Vol. 123, Issue 20). https://doi.org/10.1242/jcs.062414

Berlin, V., Styles, C. A., \& Fink, G. R. (1990). BIK1, a protein required for microtubule function during mating and mitosis in Saccharomyces cerevisiae, colocalizes with tubulin. Journal of Cell Biology, 111(6 I). https://doi.org/10.1083/jcb.111.6.2573

Branon, T. C., Bosch, J. A., Sanchez, A. D., Udeshi, N. D., Svinkina, T., Carr, S. A., Feldman, J. L., Perrimon, N., \& Ting, A. Y. (2018). Efficient proximity labeling in living cells and organisms with TurbolD. Nature Biotechnology, 36(9). https://doi.org/10.1038/nbt.4201

Britto, M., Goulet, A., Rizvi, S., Von Loeffelholz, O., Moores, C. A., \& Cross, R. A. (2016). 
Carvalho, P., Gupta, M. L., Hoyt, M. A., \& Pellman, D. (2004). Cell cycle control of kinesin-mediated transport of Bik1 (CLIP-170) regulates microtubule stability and dynein activation. Developmental Cell, 6(6). https://doi.org/10.1016/j.devcel.2004.05.001

Caudron, F., Andrieux, A., Job, D., \& Boscheron, C. (2008). A new role for kinesin-directed transport of Bik1p (CLIP-170) in Saccharomyces cerevisiae. Journal of Cell Science, 121(9). https://doi.org/10.1242/jcs.023374

Dudziak, A., Engelhard, L., Bourque, C., Klink, B. U., Rombaut, P., Kornakov, N., Jänen, K., Herzog, F., Gatsogiannis, C., \& Westermann, S. (2021). Phospho-regulated Bim1/EB1 interactions trigger Dam1c ring assembly at the budding yeast outer kinetochore. The EMBO Journal, 4O(18). https://doi.org/10.15252/embj.2021108004

Gardner, M. K., Bouck, D. C., Paliulis, L. V., Meehl, J. B., O’Toole, E. T., Haase, J., Soubry, A., Joglekar, A. P., Winey, M., Salmon, E. D., Bloom, K., \& Odde, D. J. (2008). Chromosome Congression by Kinesin-5 Motor-Mediated Disassembly of Longer Kinetochore Microtubules. Cell, 135(5). https://doi.org/10.1016/j.cell.2008.09.046

Gardner, M. K., Pearson, C. G., Sprague, B. L., Zarzar, T. R., Bloom, K., Salmon, E. D., \& Odde, D. J. (2005). Tension-dependent Regulation of Microtubule Dynamics at Kinetochores Can Explain Metaphase Congression in Yeast. Molecular Biology of the Cell, 16(8), 3764-3775. https://doi.org/10.1091/mbc.e05-04-0275

Gerson-Gurwitz, A., Thiede, C., Movshovich, N., Fridman, V., Podolskaya, M., Danieli, T., Lakämper, S., Klopfenstein, D. R., Schmidt, C. F., \& Gheber, L. (2011). Directionality of individual kinesin-5 Cin8 motors is modulated by loop 8, ionic strength and microtubule geometry. EMBO Journal, 30(24). https://doi.org/10.1038/emboj.2011.403

Hoyt, M. A., He, L., Kek Khee Loo, \& Saunders, W. S. (1992). Two Saccharomyces cerevisiae kinesinrelated gene products required for mitotic spindle assembly. Journal of Cell Biology, 118(1). https://doi.org/10.1083/jcb.118.1.109

Kops, G. J. P. L., Saurin, A. T., \& Meraldi, P. (2010). Finding the middle ground: How kinetochores 
power chromosome congression. In Cellular and Molecular Life Sciences (Vol. 67, Issue 13). https://doi.org/10.1007/s00018-010-0321-y

Kosugi, S., Hasebe, M., Tomita, M., \& Yanagawa, H. (2008). Nuclear export signal consensus sequences defined using a localization-based yeast selection system. Traffic, 9(12). https://doi.org/10.1111/j.1600-0854.2008.00825.x

Kotwaliwale, C. V., Frei, S. B., Stern, B. M., \& Biggins, S. (2007). A Pathway Containing the Ipl1/Aurora Protein Kinase and the Spindle Midzone Protein Ase1 Regulates Yeast Spindle Assembly. Developmental Cell, 13(3). https://doi.org/10.1016/j.devcel.2007.07.003 mediated by TurbolD to identify protein-protein interaction networks in yeast. Journal of Cell Science, 132(11). https://doi.org/10.1242/jcs.232249

Lin, H., De Carvalho, P., Kho, D., Tai, C. Y., Pierre, P., Fink, G. R., \& Pellman, D. (2001). Polyploids require Bik1 for kinetochore-microtubule attachment. Journal of Cell Biology, 155(7).

Longtine, M. S., McKenzie, A., Demarini, D. J., Shah, N. G., Wach, A., Brachat, A., Philippsen, P., \& Pringle, J. R. (1998). Additional modules for versatile and economical PCR-based gene deletion and modification in Saccharomyces cerevisiae. Yeast, 14(10). during mitosis. In Biology (Vol. 6, Issue 1). https://doi.org/10.3390/biology6010013 chromosomes biorient via gradual resolution of syntely between S phase and anaphase. Cell, 154(5). https://doi.org/10.1016/j.cell.2013.08.008 Protein Tagging of Microtubules in Saccharomyces cerevisiae. Traffic, 16(7). https://doi.org/10.1111/tra.12276 
Miller, R. K., D’Silva, S., Moore, J. K., \& Goodson, H. V. (2006). The CLIP-170 Orthologue Bik1p and Positioning the Mitotic Spindle in Yeast. In Current Topics in Developmental Biology (Vol. 76). https://doi.org/10.1016/S0070-2153(06)76002-1

Molk, J. N., Salmon, E. D., \& Bloom, K. (2006). Nuclear congression is driven by cytoplasmic microtubule plus end interactions in S. cerevisiae. Journal of Cell Biology, 172(1).

Moore, J. K., D'Silva, S., \& Miller, R. K. (2006). The CLIP-170 homologue Bik1p promotes the phosphorylation and asymmetric localization of Kar9p. Molecular Biology of the Cell, 17(1). https://doi.org/10.1091/mbc.E05-06-0565

O’Toole, E. T., Winey, M., \& McIntosh, J. R. (1999). High-voltage electron tomography of spindle pole bodies and early mitotic spindles in the yeast Saccharomyces cerevisiae. Molecular Biology of the Cell, 10(6). https://doi.org/10.1091/mbc.10.6.2017

Pandey, H., Reithmann, E., Goldstein-Levitin, A., Al-Bassam, J., Frey, E., \& Gheber, L. (2021). Draginduced directionality switching of kinesin-5 Cin8 revealed by cluster-motility analysis. Science Advances, 7(6). https://doi.org/10.1126/sciadv.abc1687 nanometer scale gradients in spindle microtubule dynamics using model convolution movement in the yeast Saccharomyces cerevisiae. Journal of Cell Science, 22(2). N., \& Asbury, C. L. (2009). The Ndc80 Kinetochore Complex Forms Load-Bearing Attachments to Dynamic Microtubule Tips via Biased Diffusion. Cell, 136(5). https://doi.org/10.1016/j.cell.2008.12.045 
Roostalu, J., Hentrich, C., Bieling, P., Telley, I. A., Schiebel, E., \& Surrey, T. (2011). Directional switching of the kinesin Cin8 through motor coupling. Science, 332(6025). https://doi.org/10.1126/science.1199945

Roux, K. J., Kim, D. I., Raida, M., \& Burke, B. (2012). A promiscuous biotin ligase fusion protein identifies proximal and interacting proteins in mammalian cells. Journal of Cell Biology, 196(6). https://doi.org/10.1083/jcb.201112098

Schindelin, J., Arganda-Carreras, I., Frise, E., Kaynig, V., Longair, M., Pietzsch, T., Preibisch, S., Rueden, Cardona, A. (2012). Fiji: An open-source platform for biological-image analysis. In Nature Methods (Vol. 9, Issue 7). https://doi.org/10.1038/nmeth.2019 Mechanisms of microtubule-based kinetochore positioning in the yeast metaphase spindle. Biophysical Journal, 84(6). https://doi.org/10.1016/S0006-3495(03)75087-5

Su, X., Qiu, W., Gupta, M. L., Pereira-Leal, J. B., Reck-Peterson, S. L., \& Pellman, D. (2011). Mechanisms Underlying the Dual-Mode Regulation of Microtubule Dynamics by Kip3/Kinesin-8. vivo detection of protein-protein interaction in Saccharomyces cerevisiae. Yeast, 24(9). https://doi.org/10.1002/yea.1504 the kinetochore couples microtubule force and centromere stretch to move chromosomes. Nature Cell Biology, 18(4). https://doi.org/10.1038/ncb3323 A Kinesin-5, Cin8, Recruits Protein Phosphatase 1 to Kinetochores and Regulates Chromosome Segregation. Current Biology, 28(17). https://doi.org/10.1016/j.cub.2018.08.038

491 Tytell, J. D., \& Sorger, P. K. (2006). Analysis of kinesin motor function at budding yeast kinetochores. 
Journal of Cell Biology, 172(6). https://doi.org/10.1083/jcb.200509101

493 Varga, V., Helenius, J., Tanaka, K., Hyman, A. A., Tanaka, T. U., \& Howard, J. (2006). Yeast kinesin-8

494 depolymerizes microtubules in a length-dependent manner. Nature Cell Biology, 8(9). https://doi.org/10.1038/ncb1462

Wargacki, M. M., Tay, J. C., Muller, E. G., Asbury, C. L., \& Davis, T. N. (2010). Kip3, the yeast kinesin-8, is required for clustering of kinetochores at metaphase. Cell Cycle, 9(13). https://doi.org/10.4161/cc.9.13.12076

Winey, M., Mamay, C. L., O’Toole, E. T., Mastronarde, D. N., Giddings, T. H., McDonald, K. L., \& McIntosh, J. R. (1995). Three-dimensional ultrastructural analysis of the Saccharomyces cerevisiae mitotic spindle. Journal of Cell Biology, 129(6). https://doi.org/10.1083/jcb.129.6.1601

Winey, M., \& O’Toole, E. T. (2001). The spindle cycle in budding yeast. In Nature Cell Biology (Vol. 3, Issue 1). https://doi.org/10.1038/35050663 https://doi.org/10.1016/j.semcdb.2017.10.024 
$\mathbf{A}$

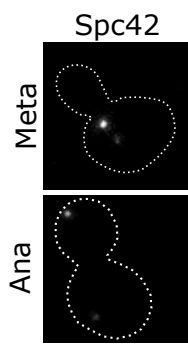

Bik1

Tub1

Spc42/Bik1/Tub1

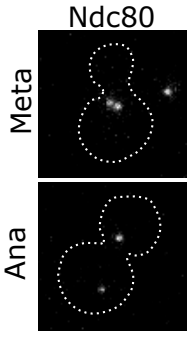

Bik1
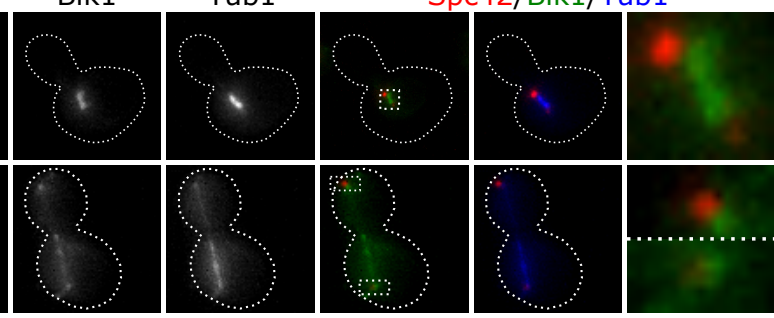

B
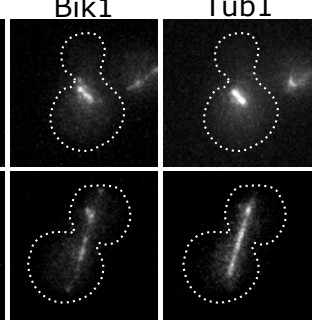

Ndc80/Bik1/Tub1
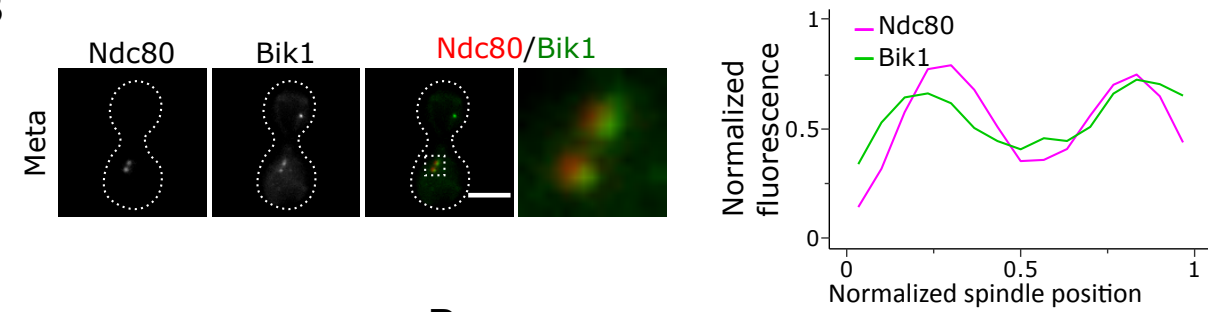

C

D
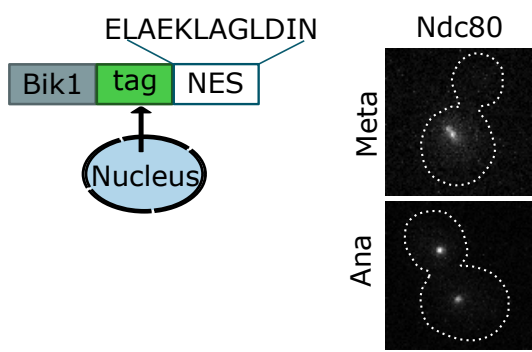

Bik1

Tub1 Ndc80/Bik1/Tub1
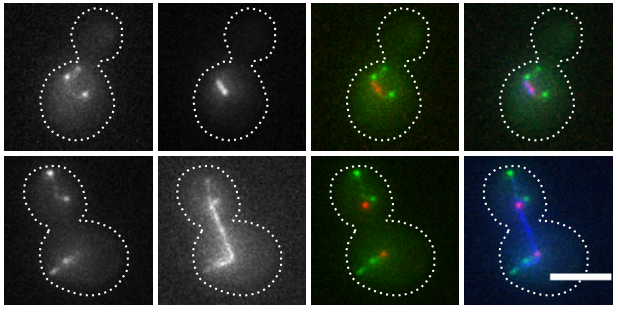

E
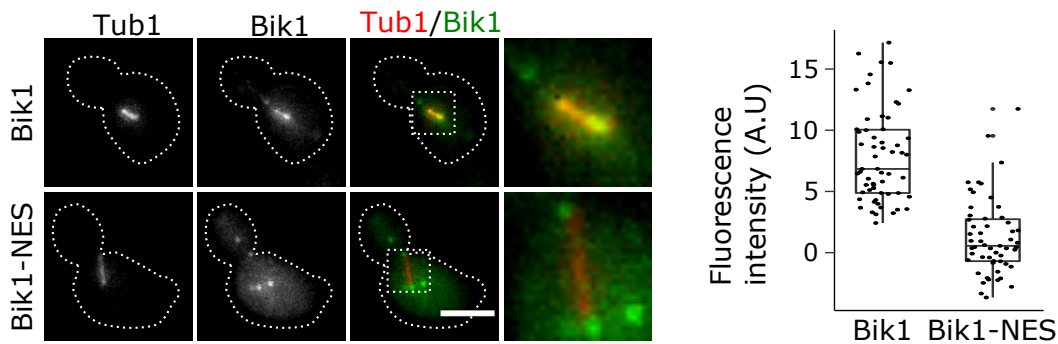

Figure 1. Bik1 localizes to kinetochores in a cell-cycle dependent manner. (A) Representative images of Bik1-sfGFP (green) localization in cycling cells expressing mTurquoise-Tub1 (blue) and Spc42-tagRFP-T (red, top panel), and Nc80-tagRFP-T (red, lower panel). Dashed boxes show area enlarged in the rightmost images. (B) Left panel: Representative images of Bik1-sfGFP (green) localization in pGal1-Cdc20 metaphase-arrested cells expressing Nc80-tagRFP-T. Right panel: Line scan analysis shows the distribution of Bik1-sfGFP and kinetochores (Ndc80-tagRFP-T) in metaphase arrested cells. For each cell, the fluorescence intensity was measured along a line drawn between the kinetochore foci and normalized relative to the maximum value, and the mean values are shown ( $n=26$ cells). Dashed boxes show area enlarged in the rightmost images. (C) Cartoon showing the design of Bik1-NES. In short, a strong nuclear export signal (NES) was fused to Bik1-sfGFP or Bik1-5xFlag to promote nuclear export. (D) Representative images of Bik1-sfGFP-NES (green) localization in cycling cells expressing mTurquoise-Tub1 (blue) and Ndc80-tagRFPT (red). (E) Quantification of Bik1-sfGFP or Bik1-sfGFP-NES localization in metaphase cells. Bik1-sfGFP and Bik1-sfGFP-NES cells expressing mRuby2-Tub1 were arrested in G1 with $\alpha$-factor and subsequently released in fresh media. Cells were fixed every 10 minutes for 120 minutes. Left: Representative cells at metaphase. Time after release $=40$ min (Bik1sfGFP), and $50 \mathrm{~min}$ (Bik1-sfGFP-NES). Right: Boxplots of the integrated fluorescence intensity of Bik1-sfGFP and Bik1-sfGFP-NES at the spindle (mRuby2-Tub1) in metaphase cells ( $n=59$ cells for Bik1-sfGFP and 55 for Bik1-sfGFP-NES). Dashed boxes show area enlarged in the rightmost images. All images shown are maximum intensity projections of Z-stacks ( $9 \mu \mathrm{m}, 1 \mu \mathrm{m}$ steps). Dashed lines represent the cell outlines based on bright field images. All scale bars, $4 \mu \mathrm{m}$. 
bioRxiv preprint doi: https://doi.org/10.1101/2021.06.16.447861; this version posted October 14, 2021. The copyright holder for this preprint (which was not certified by peer review) is the author/funder. All rights reserved. No reuse allowed without permission.

A
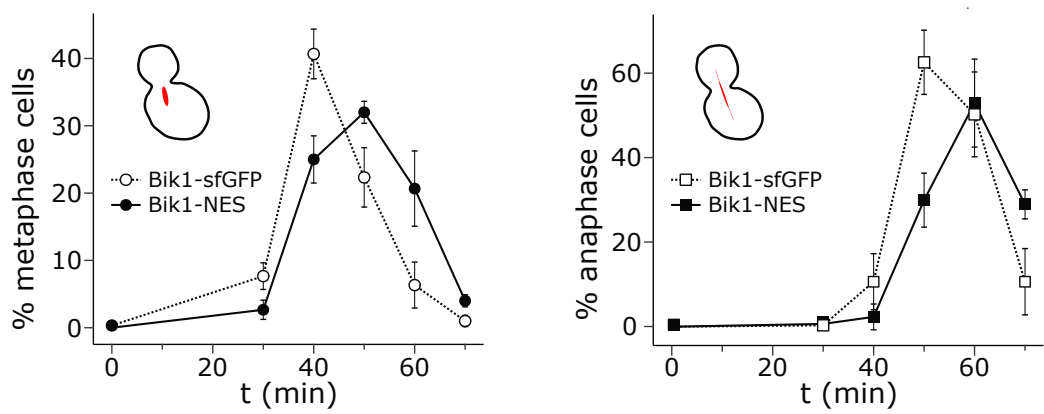

B
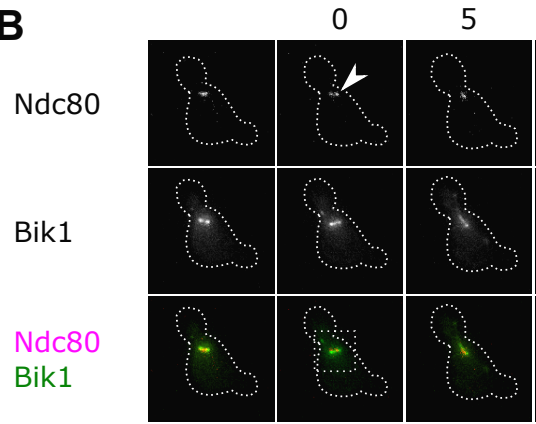

10
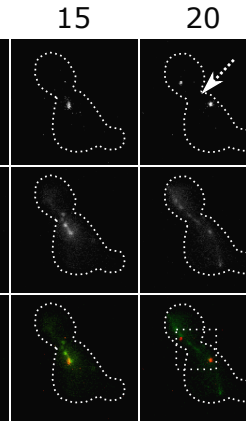

25
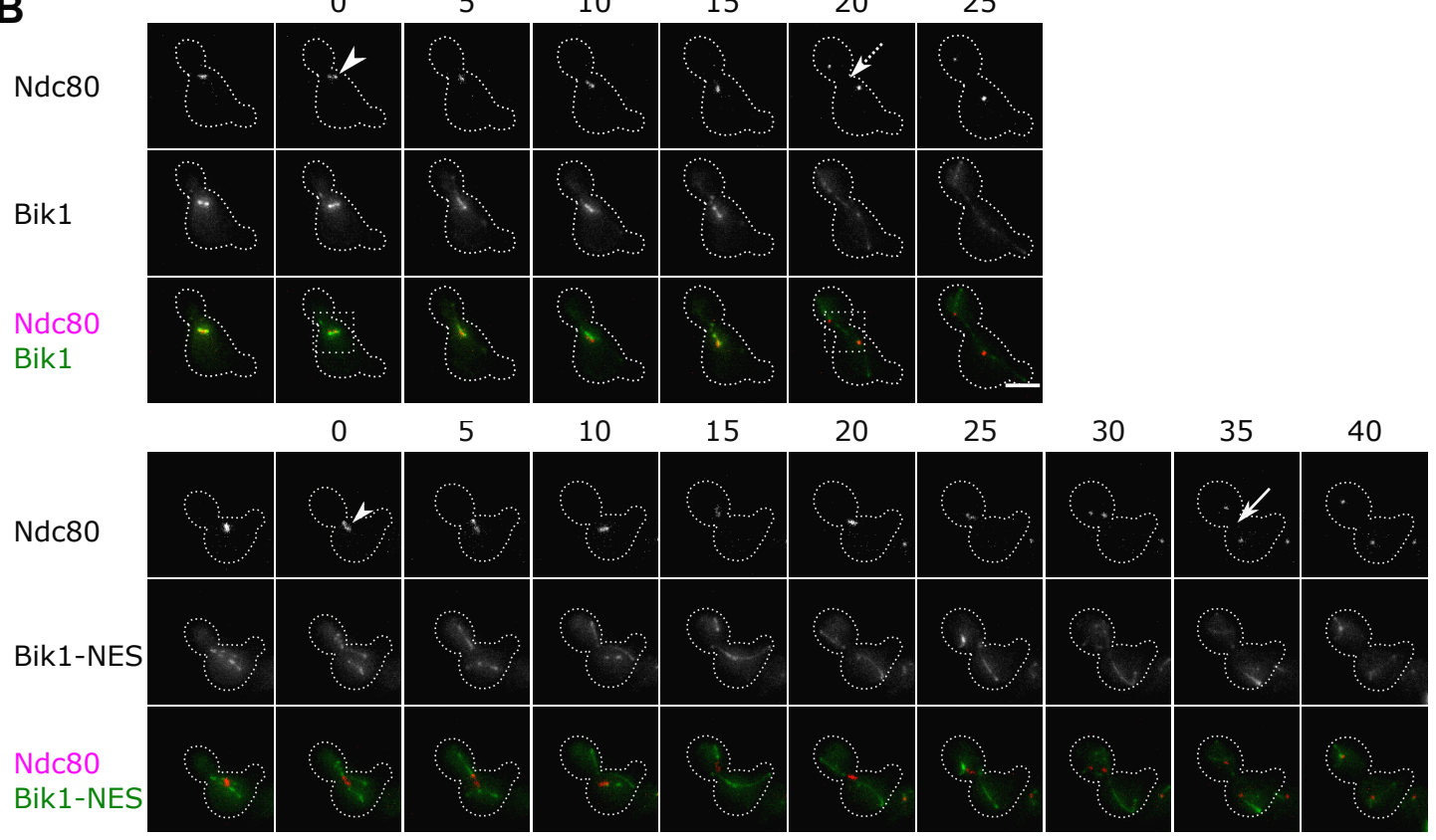

C

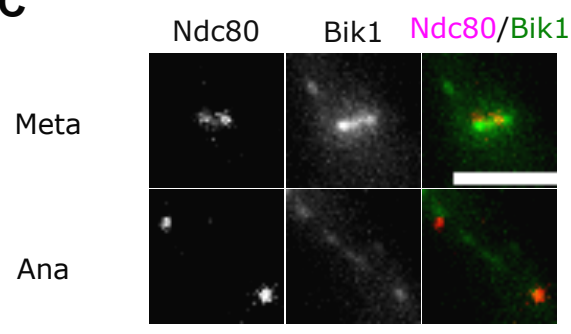

D

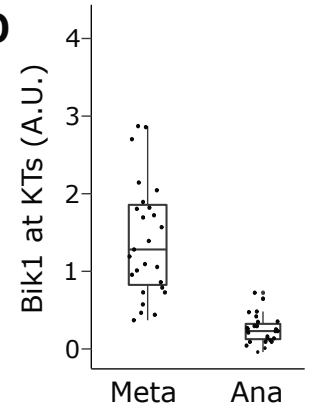

$\mathbf{E}$

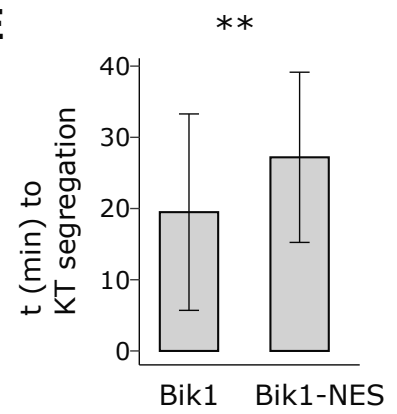

Figure 2. Bik1-NES delays spindle elongation at metaphase/anaphase transition. (A) Cells from (1E) were scored for cell cycle progression based on bud size and spindle length (mRuby-Tub1). Error bars represent mean $\pm s d(n=3$ independent experiments, each experiment with 100 cells each timepoint for each strain (Bik1-sfGFP and Bik1-sfGFP-NES) analysed per experiment). (B-E) Time-lapse imaging of cells expressing Bik1-sfGFP or Bik1-sfGFP-NES (green) and Ndc80-tagRFP-T (red). Cells were arrested in G1 with $\alpha$-factor and subsequently released in fresh medium under the microscope. Images were taken at 5 min intervals. (B) Representative time-lapse images. Arrowheads indicate the appearance of separated kinetochore clusters. Arrows indicate segregated kinetochores in mother/bud. The time ( $\mathrm{min}$ ) elapsed from appearance of separated kinetochore clusters is indicated by the numbers above the frames. Dashed boxes show area enlarged in (C). Images shown are maximum intensity projections of Z-stacks ( $9 \mu \mathrm{m}, 1 \mu \mathrm{m}$ steps). Scale bar, $4 \mu \mathrm{m}$. (C) Zoomed-in images from the dashed areas of (B), showing the kinetochores (Ndc80-tagRFP-T) at metaphase and anaphase. (D) Boxplots of the integrated Bik1sfGFP fluorescence intensity at kinetochores (Ndc80-tagRFP-T) at metaphase (meta) and anaphase (ana) ( $n=27$ cells). (E) Quantification of average time from the appearance of separated kinetochore clusters to segregated kinetochores in mother/bud in Bik1-sfGFP and Bik1sfGFP-NES cells. We performed two independent experiments and pooled the data. Error bars represent mean $\pm s d(n=59$ cells for Bik1sfGFP and 57 for Bik1-sfGFP-NES, p-value $=0.001691$ using Welch Two Sample t-test (two-sided)). 
bioRxiv preprint doi: https://doi.org/10.1101/2021.06.16.447861; this version posted October 14, 2021. The copyright holder for this preprint (which was not certified by peer review) is the author/funder. All rights reserved. No reuse allowed without permission.

A

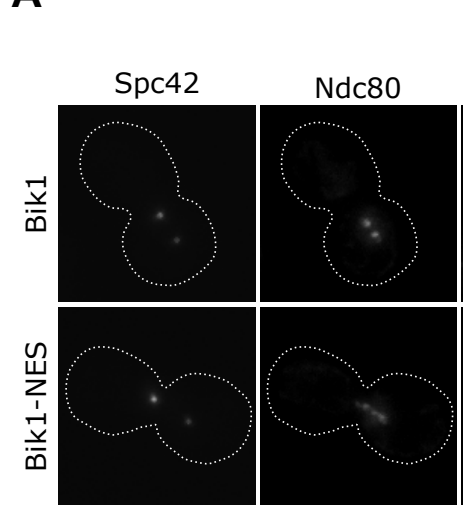

B
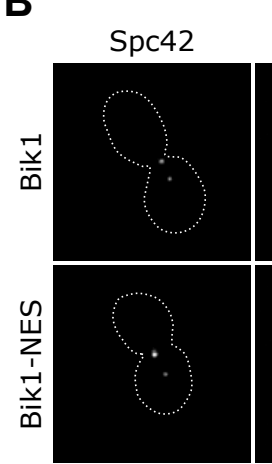

Ndc80

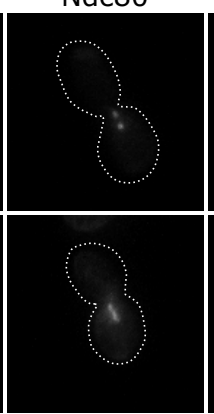

Spc42

Ndc80

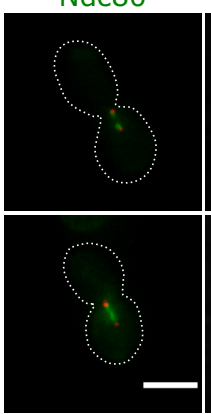

$\operatorname{Spc} 42$

Ndc80
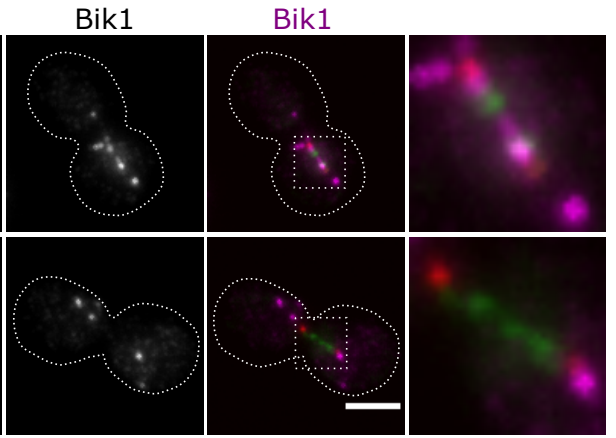

C

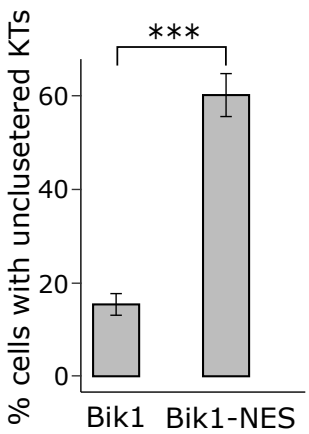

D

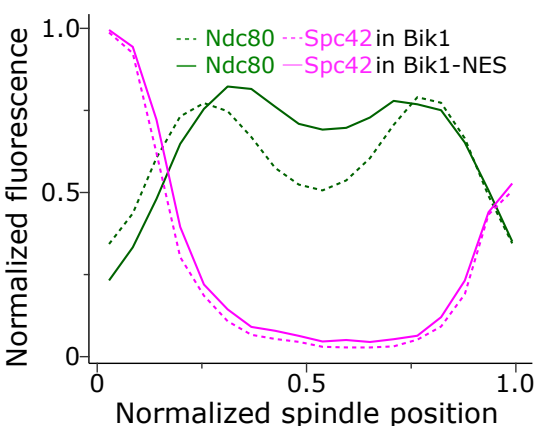

Figure 3. Nuclear Bik1 is needed for kinetochore clustering at metaphase. (A) Representative immunofluorescence staining for Bik1-5xFlag and Bik1-5xFlag-NES (magenta) in metaphase arrested pGal1-Cdc20 cells expressing Spc42-sfGFP (red) and Ndc80-TagRFP-T (green). Images shown are maximum intensity projections of Z-stacks ( $4.95 \mu \mathrm{m}, 0.3 \mu \mathrm{m}$ steps). Dashed boxes show area enlarged in the rightmost images. Scale bar, $4 \mu \mathrm{m}$. (B) Representative images of Bik1-5xFlag and Bik1-5xFlag-NES arrested as in (A), including zoomed-in images of the kinetochores (Ndc80-sfGFP). Images shown are maximum intensity projections of Z-stacks (8.7 $\mu \mathrm{m}, 0.3 \mu \mathrm{m}$ steps). Scale bar, $4 \mu \mathrm{m}$. (C) Quantification of kinetochore (Ndc80-tagRFPT) unclustered phenotype in Bik1-5xFlag and Bik-5xFlag-NES cells arrested in metaphase as shown in (B). Error bars represent mean $\pm s d$ ( $n=3$ independent experiments, with 100 cells for each strain (Bik1-5xFlag and Bik1-5xFlagNES). $p=0.0006884$ using Welch Two Sample t-test (two-sided). (D) Line scan analysis shows the distribution of kinetochores (Ndc80sfGFP) and spindle pole bodies (SPBs, Spc42-tagRFP-T) in metaphase arrested cells. For each cell, the fluorescence intensity was measured along lines drawn between the SPBs (Spc42-TagRFP-T) and normalized relative to the maximum value. Mean values are shown ( $\mathrm{n}=100$ cells for each strain (Bik1-5xFlag and Bik1-5xFlag-NES). 
bioRxiv preprint doi: https://doi.org/10.1101/2021.06.16.447861; this version posted October 14, 2021. The copyright holder for this preprint (which was not certified by peer review) is the author/funder. All rights reserved. No reuse allowed without permission.

A

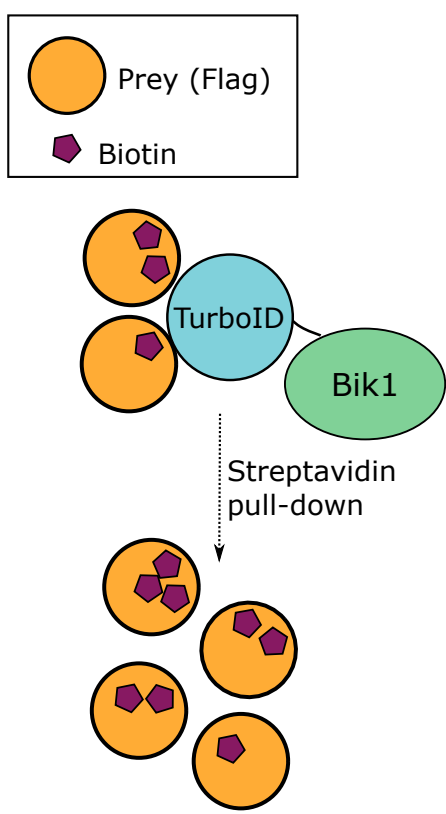

B
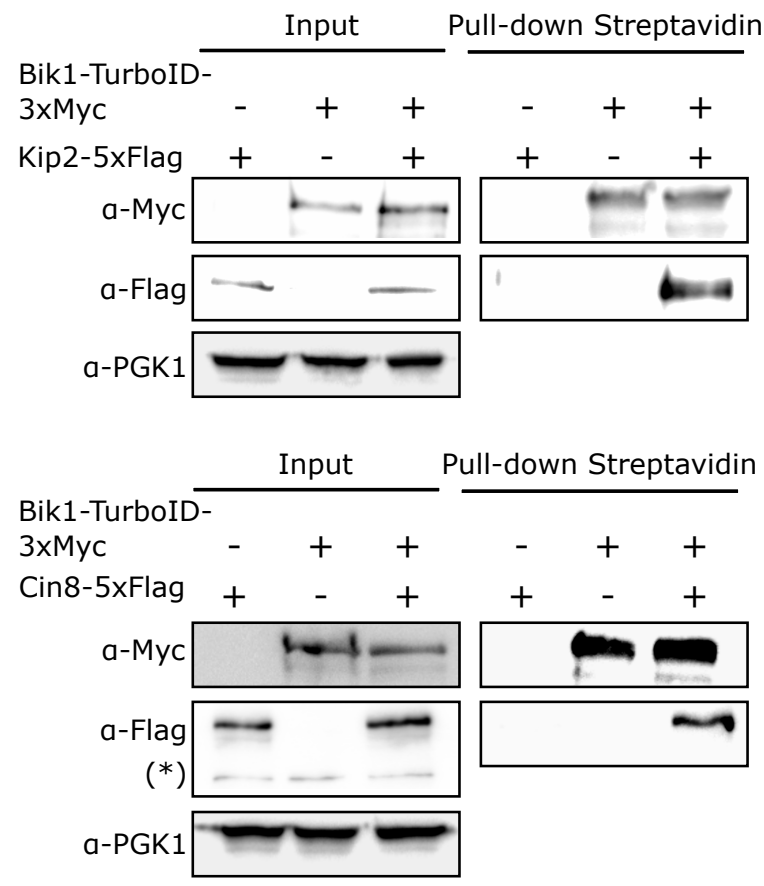

C

Cin8-Bik1

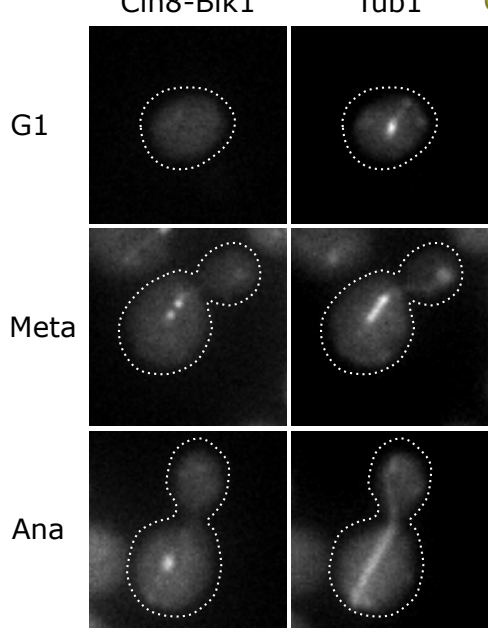

Cin8-Bik1/Tub1

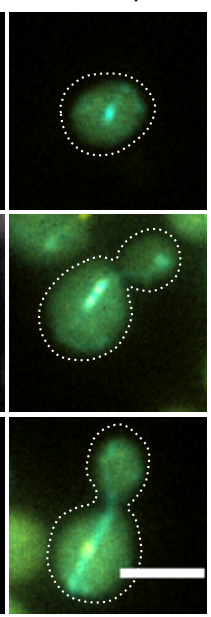

D

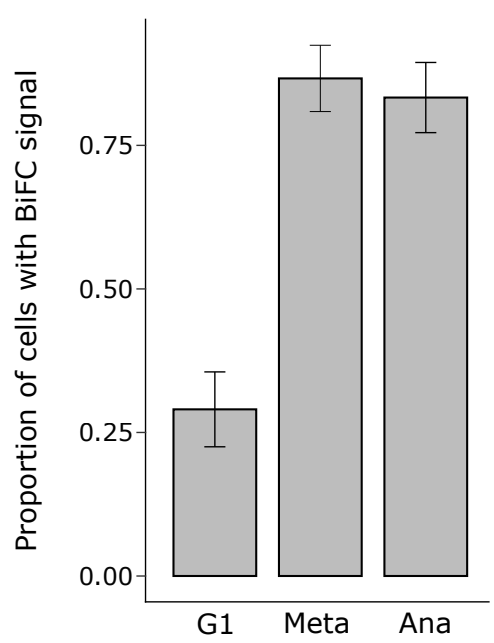

Figure 4. Bik1 interacts with Cin8 at metaphase. (A-B) Identification of Cin8 as a Bik1 interaction partner using TurbolD. Cartoon outlining the principle of the TurbolD assay. Briefly, a biotin ligase was fused to endogenous Bik1 (Bik1-TurbolD) in cells expressing the protein of interest tagged with Flag (Prey). Biotinylated substrates were purified using streptavidin beads, and inputs and pull-down lysates were analyzed by immunoblot for the indicated proteins. (B) Western blot analysis of total cell extracts (input) and streptavidin pull-downs prepared from Bik1-TurbolD-3xmyc cells expressing Kip2-5xFlag or Cin8-5xFlag as prey, and control strains, using anti-Myc (top) and antiFlag (bottom) antibodies. (*) unspecific band. (C-D) Bimolecular fluorescence complementation (BiFC) analysis of Bik1 and Cin8 interaction. (C) Representative images of cycling cells expressing Bik1-VN and Cin8-VC in G1, metaphase and anaphase. Images shown are single focal planes. (D) Proportion of cells in (C) with BIFC signal based on Venus fluorescence. Error bars represent mean $\pm s d(n=3$ independent experiments, with 50 cells analyzed in each cell cycle phase (G1, metaphase and anaphase) per experiment. 
bioRxiv preprint doi: https://doi.org/10.1101/2021.06.16.447861; this version posted October 14, 2021. The copyright holder for this preprint (which was not certified by peer review) is the author/funder. All rights reserved. No reuse allowed without permission.

A
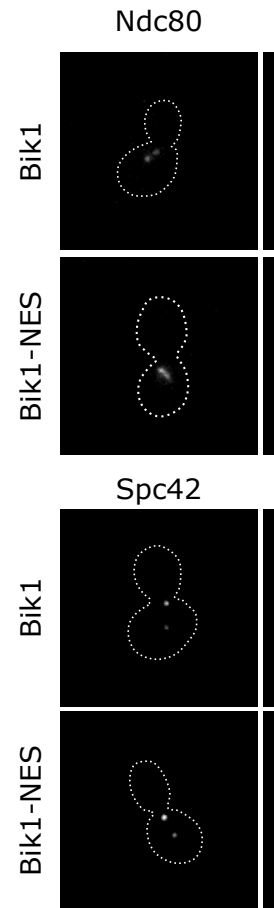

C

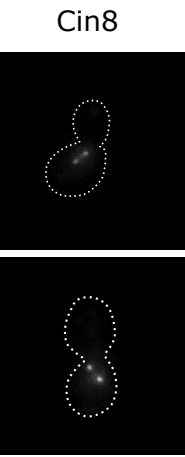

Cin8

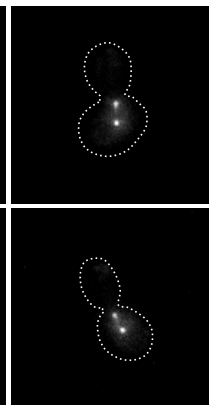

Bik1 Ndc80/Cin8

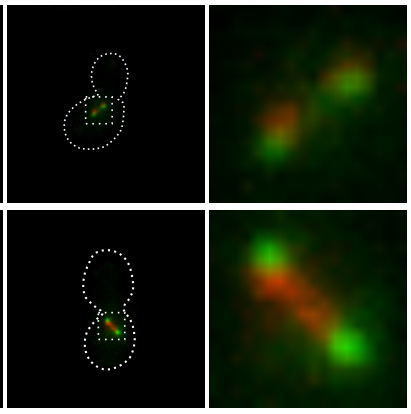

Spc42/Cin8
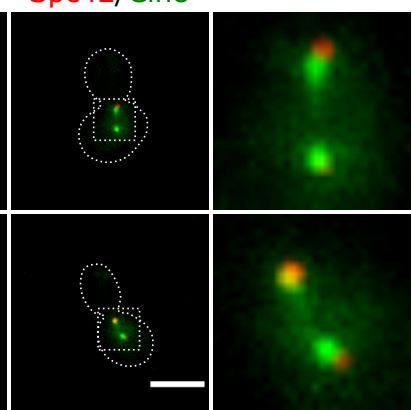

Ndc80/Bik1
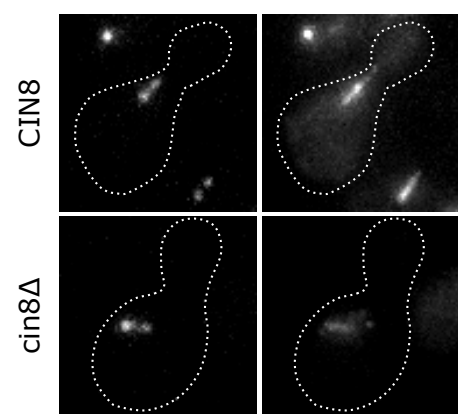

B

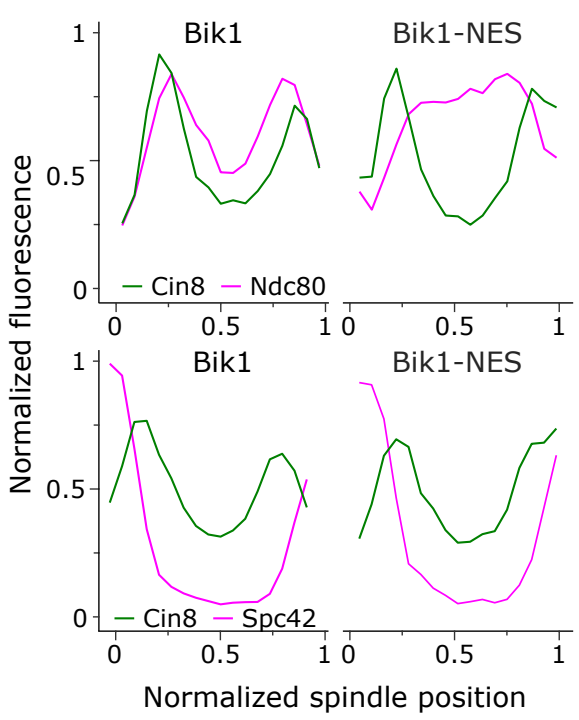

D

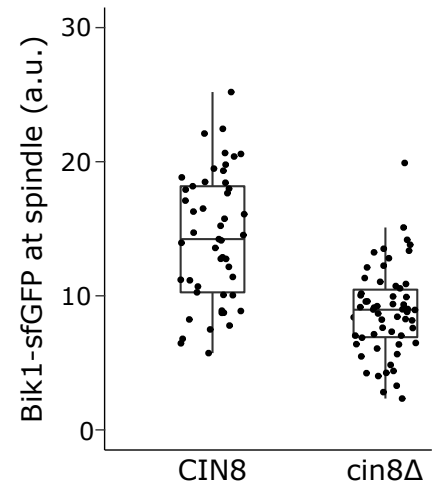

Figure 5. Dependence of Cin8 and Bik1 to localize to the spindle. (A-B) Cin8 localizes at the spindle in Bik1-NES cells but does not co-localize with unclustered kinetochores. (A) Cin8 localization at the metaphase spindle does not require nuclear Bik1. Representative images of pGal1-Cdc20 cells arrested in metaphase expressing Bik1-5xFlag or Bik1-5xFlag-NES, Cin8-sfGFP, and the markers Ndc80-TagRFP-T (top panel) or Spc42-TagRFP-T (lower panel). Images shown are maximum intensity projections of Z-stacks (8.7 $\mu \mathrm{m}, 0.3 \mu \mathrm{m}$ steps). Dashed boxes show area enlarged in the rightmost images. Scale bar, $4 \mu \mathrm{m}$. (B) Line scan analysis of Cin8 distribution (Cin8-sfGFP) in metaphase arrested cells). Top panel: Cin8 distribution relative to the kinetochores (Ndc80-tagRFP-T). For each cell, the fluorescence intensities of Cin8-sfGFP and Ndc80-tagRFPT was measured along lines drawn between the SPBs and normalized relative to the maximum values, mean values are shown ( $n=25$ cells for BIK1 and 25 for Bik1-5xFlag-NES). Low panel: Cin8 distribution relative to the SPBs (Spc42-tagRFP-T). For each cell, the fluorescence intensity of Cin8-sfGFP and Spc42-tagRFPT was measured along a line drawn between the SPBs and normalized relative to the maximum value. Mean values are shown ( $n=25$ cells for Bik1-5xFlag and 26 for Bik1-5xFlag-NES). (C) Cin8 is needed for Bik1-sfGFP localization at kinetochores. CIN8 and cin8 $\Delta$ cells expressing Bik1-sfGFP and Ndc80-TagRFP-T were arrested in G1 using $\alpha$-factor, released in fresh media at $25^{\circ} \mathrm{C}$ and fixed every 10 minutes for 130 minutes. Left: Representative images of cells in metaphase (CIN8, 60 min after release; cin8 $8,110 \mathrm{~min}$ after release). Images shown are maximum intensity projections of Z-stacks (6.6 $\mu \mathrm{m}, 0.6 \mu \mathrm{m}$ steps). Dashed boxes show area enlarged in the rightmost images. Scale bar, $4 \mu \mathrm{m}$.

(D) Boxplots of the integrated fluorescence intensity of Bik1-sfGFP at the spindle (based on Ndc80-TagRFP-T localization) in metaphase cells ( $\mathrm{n}=100$ cells for each strain (CIN8 and $\operatorname{cin} 8 \Delta)$. 
Table 1. A subset of the protein interactors of Bik1-FLAG (See Data S1 for a complete list).

\begin{tabular}{|l|r|r|}
\hline Name & \% Coverage & $\begin{array}{l}\text { Unique } \\
\text { peptides }\end{array}$ \\
\hline STU2 & 72.33 & 104 \\
\hline BIM1 & 67.00 & 21 \\
\hline CIN8 & 50.67 & 46 \\
\hline KIP2 & 49.33 & 28 \\
\hline KAR9 & 47.33 & 26 \\
\hline KIP1 & 38.67 & 28 \\
\hline KAR3 & 38.67 & 23 \\
\hline CIK1 & 22.67 & 10 \\
\hline VIK1 & 21.00 & 10 \\
\hline KIP3 & 15.33 & 6 \\
\hline
\end{tabular}


Table S1. Strains used in this study. All strains are derived from BY4741

\begin{tabular}{|l|l|}
\hline Strain & Genotype \\
\hline VMB-212 & MAT $\alpha$ BIK1-sfGFP:Ura3 SPC42-tagRFP-T:KanMX Leu2:HIS3p:mTurquoise2-TUB1 \\
\hline VMB-154 & MATa BIK1-sfGFP:Ura3 NDC80-tagRFP-T:KanMX Leu2:HIS3p:mTurquoise2-TUB1 \\
\hline VMB-185 & MATa BIK1-sfGFP:Ura3 NDC80-tagRFP-T:KanMX \\
\hline VMB-293 & MATa NatN2:PGAL1-CDC20 BIK1-sfGFP:Ura3 NDC80-tagRFP-T:KanMX \\
\hline VMB-156 & $\begin{array}{l}\text { MAT } \alpha \text { BIK1-sfGFP-NES:NatMX NDC80-tagRFP-T:KanMX Leu2:HIS3p:mTurquoise2- } \\
\text { TUB1 }\end{array}$ \\
\hline VMB-166 & MATa BIK1-sfGFP:Ura3 Leu2:HIS3p:mRuby2-TUB1 \\
\hline VMB-209 & MATa BIK1-sfGFP-NES:NatMX Leu2:HIS3p:mRuby2-TUB1 \\
\hline VMB-455 & $\begin{array}{l}\text { MATa HygroMX:PGAL1-CDC20 BIK1-5xFlag-NES:NatMX NDC80-sfGFP:Ura3 SPC42- } \\
\text { tagRFP-T:KanMX }\end{array}$ \\
\hline VMB-458 & $\begin{array}{l}\text { MATa HygroMX:PGAL1-CDC20 BIK1-5xFlag:NatMX NDC80-sfGFP:Ura3 SPC42-tagRFP- } \\
\text { T:KanMX }\end{array}$ \\
\hline VMB-433 & MATa KIP2-5xFlag:KanMX \\
\hline VMB-422 & MATa BIK1-TurboID-3xMyc:NatMX \\
\hline VMB-431 & MATa BIK1-TurboID-3xMyc:NatMX KIP2-5xFlag:KanMX \\
\hline VMB-393 & MATa CIN8-5xFlag:KanMX \\
\hline VMB-428 & MATa BIK1-TurboID-3xMyc:NatMX CIN8-5xFlag:KanMX \\
\hline VMB-448 & MATa HygroMX:PGAL1-CDC20 CIN8-sfGFP:Ura3 NDC80-tagRFP-T:KanMX \\
\hline VMB-453 & $\begin{array}{l}\text { MATa HygroMX:PGAL1-CDC20 BIK1-5xFlag-NES:NatMX CIN8-sfGFP:Ura3 NDC80- } \\
\text { tagRFP-T:KanMX }\end{array}$ \\
\hline VMB-459 & $\begin{array}{l}\text { MATa HygroMX:PGAL1-CDC20 BIK1-5xFlag:NatMX CIN8-sfGFP:Ura3 SPC42-tagRFP- } \\
\text { T:KanMX }\end{array}$ \\
\hline VMB-457 & $\begin{array}{l}\text { MATa HygroMX:PGAL1-CDC20 BIK1-5xFlag-NES:NatMX CIN8-sfGFP:Ura3 SPC42- } \\
\text { tagRFP-T:KanMX }\end{array}$ \\
\hline VMB-308 & MATa BIK1-sfGFP:Ura3 cin8D:natMX NDC80-tagRFP-T:KanMX \\
\hline VMB-288 & MATa NatN2:PGAL1-CDC20 BIK1-5xFlag:KanMX \\
\hline VMB-494 & MATa BIK1-VN173:His3MX CIN8-VC155:KanMX Leu2:HIS3p:mTurquoise2-TUB1 \\
\hline
\end{tabular}


Table S2. Plasmids used in this study

\begin{tabular}{|l|l|l|}
\hline Plasmid & Relevant insert & Source \\
\hline p44873 & $\begin{array}{l}\text { pFA6a-link-yosuperfolderGFP- } \\
\text { CaURA3 }\end{array}$ & $\begin{array}{l}\text { Gift from Wendell Lim \& Kurt Thorn } \\
\text { (Addgene: 44873) PMID: 23844123 }\end{array}$ \\
\hline p44906 & pFA6a-link-yoTagRFP-T-Kan & $\begin{array}{l}\text { Gift from Wendell Lim \& Kurt Thorn } \\
\text { (Addgene: 44906) PMID: 23844123 }\end{array}$ \\
\hline p50641 & $\begin{array}{l}\text { pHIS3p:mTurquoise2- } \\
\text { Tub1+3'UTR::LEU2 }\end{array}$ & $\begin{array}{l}\text { Gift from Wei-Lih Lee } \\
\text { (Addgene: 50641) PMID: 25711127 }\end{array}$ \\
\hline p50645 & $\begin{array}{l}\text { pHIS3p:mRuby2- } \\
\text { Tub1+3'UTR::LEU2 }\end{array}$ & $\begin{array}{l}\text { Gift from Wei-Lih Lee } \\
\text { (Addgene: 50645) PMID: 25711127 }\end{array}$ \\
\hline pYM-N23 & $\begin{array}{l}\text { ScGAL1 promoter::pAgTEF- } \\
\text { nat-tScADH1 }\end{array}$ & $\begin{array}{l}\text { PCR-Toolbox Knop } \\
\text { (Euroscarf) MID: 15334558 }\end{array}$ \\
\hline p15983 & $\begin{array}{l}\text { pFA6a-5xFlag-KanMX6 } \\
\text { pFB1434 }\end{array}$ & $\begin{array}{l}\text { Gift from Eishi Noguchi } \\
\text { (Addgene: 15983) PMID: 18729046 }\end{array}$ \\
\hline P30555 & $\begin{array}{l}\text { pFA6a-5xFlag-NatMX6 } \\
\text { NatMX6 }\end{array}$ & This study (subclonned from p15983) \\
\hline P30560 & pFA6a-VN-His3MX6 & $\begin{array}{l}\text { Gift from François Bachand } \\
\text { (Addgene: 126050) PMID: 31064814 }\end{array}$ \\
\hline & pFA6a-VC-kanMX6 & $\begin{array}{l}\text { Gift from Sung MK \& Huh WK. } \\
\text { (Euroscarf) PMID: 17534848 }\end{array}$ \\
\hline
\end{tabular}

